\title{
LIMITAÇÕES E PROBLEMAS NO TRANSPORTE DA SOJA NO BRASIL
}

Limitations and problems in soy transport in Brazil

DOI: 10.48075/igepec.v25i1.25650

Maicon Gonçalves Monteiro

Marlon Vinícius Brisola

Fabrício Oliveira Leitão

Warley Henrique da Silva 


\title{
Limitações e Problemas no Transporte da Soja no Brasil
}

\author{
Limitations and problems in soy transport in Brazil
}

\author{
DOI: 10.48075/igepec.v25i1.25650 \\ Maicon Gonçalves Monteiro \\ Marlon Vinícius Brisola \\ Fabrício Oliveira Leitão \\ Warley Henrique da Silva
}

\begin{abstract}
Resumo: O Brasil, maior produtor e exportador de soja do mundo, ainda enfrenta sérios problemas no transporte da soja. Assim, o objetivo do estudo foi identificar as limitações e os principais problemas do transporte dessa oleaginosa, destacando as principais consequências entre 2000 e 2019. O estudo é exploratório, descritivo e qualitativo, e utilizada da revisão sistemática da literatura seguindo o protocolo de Cronin et. al. (2008). Os principais achados relacionam-se à falta de estruturas de armazéns e terminais intermodais, estradas degradadas, hidrovias sem acesso direto aos portos e com trechos não navegáveis, linhas férreas com bitolas diferentes e com extensão limitada, más condições dos veículos e um sistema deficiente de balanças para pesagem da soja que segue aos portos saindo da região centro-oeste. Os resultados direcionam questões de estudo relacionadas ao grau de influência desses problemas na produção de soja e as dificuldades de estabelecer Parcerias Público-Privado para resolução dos gargalos.
\end{abstract}

Palavras-chaves: Logística, Transporte, Soja, Revisão Sistemática de Literatura.

\begin{abstract}
Brazil, the world's largest soybean producer and exporter, still faces serious problems in transporting soybeans. Thus, the objective of the study was to identify the limitations and the main problems of the transportation of this oilseed, highlighting the main consequences between 2000 and 2019. The study is exploratory, descriptive and qualitative, and used the systematic review of the literature following the protocol of Cronin et. al. (2008). The main findings are related to the lack of warehouse structures and intermodal terminals, degraded roads, waterways without direct access to ports and with non-navigable stretches, railway lines with different gauges and with limited length, poor vehicle conditions and a deficient system of scales for weighing soy that goes to ports leaving the midwest region. The results address study questions related to the degree of influence of these problems on soybean production and the difficulties of establishing Public-Private Partnerships to resolve bottlenecks.
\end{abstract}

Key words: Logistics, Transport, Soy, Systematic Literature Review.

Resumen: Brasil, el mayor productor y exportador de soja del mundo, todavía enfrenta serios problemas en el transporte de soja. Así, el objetivo del estudio fue identificar las limitaciones y los principales problemas del transporte de esta oleaginosa, destacando las principales consecuencias entre 200o y 2019. El estudio es exploratorio, descriptivo y cualitativo, y utilizó la revisión sistemática de la literatura siguiendo el protocolo de Cronin et. al. (2008). Los principales hallazgos están relacionados con la falta de estructuras de almacén y terminales intermodales, carreteras degradadas, vías navegables sin acceso directo a puertos y con tramos no navegables, líneas ferroviarias de diferente ancho y longitud limitada, malas condiciones de los vehículos y un sistema deficiente de balanzas para pesar soja que va a puertos que salen de la región centro-oeste. Los resultados abordan cuestiones del estudio relacionadas con el grado de influencia de estos problemas en la producción de soja y las dificultades de establecer Asociaciones Público-Privadas para resolver cuellos de botella.

Palabras-claves: Logística, Transporte, Soja, Revisión sistemática de la literatura. 
Informe GEPEC, ISSN:1679-415X, Toledo, V. 25, n.1, p.261-283, jan./jun. 2021. 


\section{INTRODUÇÃO}

No Brasil, o agronegócio se tornou um dos principais setores que impulsionam a economia do País, tendo expressiva composição no seu Produto Interno Bruto - PIB. O Centro de Estudos Avançados em Economia Aplicada - CEPEA (2020) indica o ramo agrícola como o grande responsável por esse resultado, participando com aproximadamente 14,6\%, em 2019, dos 20,9\% atribuído ao agronegócio no geral.

Com a safra de 2019/2020 acima dos 123 milhões de toneladas de soja colhida, o Brasil volta a liderar o ranking dos países produtores de soja como maior produtor mundial, conforme levantado pelo United States Department of Agriculture - USDA (2020). Mas o Brasil ainda enfrenta sérios problemas de infraestrutura logística, o que prejudica fortemente o escoamento da produção do grão, principalmente pela precariedade de sua matriz de transporte, especialmente quando considerado os principais modais de transporte.

Souza et. al. (2016) enfatizaram em seu trabalho o quanto as infraestruturas logísticas das principais rotas brasileiras de escoamento são limitadas, prejudicando o uso de mais de um modal e, segundo Barboza (2014), a redução dos custos logísticos que são muito elevados. Melo et. al. (2018) corroboram dizendo que a excessiva dependência do modal rodoviário pelo Brasil favorece a ineficiência de suas rotas de escoamento, principalmente quando comparadas com rotas de países como o Estados Unidos, o qual possui rotas consideradas bem eficientes, sendo que, segundo Gudolle et. al. (2019), essa ineficiência das rotas no Brasil, juntamente com a grande utilização do modal rodoviário, estão a falta de estradas em boas condições, de armazéns e portos com tecnologias modernas. Filardo et. al. (2005) também destacaram a falta de modernização.

Assim, é importante entender que logística representa o conjunto de atividades de movimentação e armazenagem que facilitam o fluxo de produtos e serviços desde sua origem até o mercado consumidor, incluindo o fluxo de informações e a manutenção dos níveis de serviços, que devem ser realizados a um custo aceitável para o cliente (CAUDURO, LEMOS; 2010). No que diz respeito as atividades primárias da logística, elas são basicamente três: transporte, manutenção de estoque e processamento de pedidos (BALLOU, 1993), as quais além de constituírem a maior parcela dos custos logísticos totais em empresas de diferente porte, ainda são muito importantes para que os clientes recebam os produtos e serviços onde e quando desejarem e na quantidade acordada (ALMEIDA, 2020).

E o transporte como um componente logístico é considerado um elementochave para um bom desempenho competitivo de uma cadeia de suprimentos, principalmente no setor agrícola, envolvendo a movimentação de matéria-prima e de produtos semiacabados ou acabados de uma origem até o mercado consumidor (CAIXETA FILHO, 2010). Assim, vale ressaltar que as condições estruturais de escoamento da produção agrícola de um determinado país influenciam na competitividade de seus produtos e, consequentemente, no seu desenvolvimento (PETROV; TURYGIN, 2011).

Vários autores buscaram estudar questões relacionadas ao transporte no escoamento da soja e de outras commodities, e dentre outros, alguns exemplos se destacam na literatura.

Pontes et. al. (2009) realizaram um levantamento dos principais estudos voltadas aos problemas logísticos de exportação da soja brasileira, todavia, a metodologia utilizada não envolve uma revisão sistêmica da literatura e nem um 
período específico de análise; Correa e Ramos (2010) focaram seu trabalho no transporte rodoviário e no seu estado precário em detrimento de outros modais de transporte daquela soja em grão proveniente apenas da região Centro-Oeste brasileira; Gaban e Guarnieri (2015) pesquisaram na literatura os principais entraves e barreiras existentes no campo da logística agroindustrial de diferentes produtos por meio de uma revisão sistemática de literatura em bases de dados nacionais e internacionais; Santos e Pereira (2019) buscaram analisar as articulações espaciais da organização territorial da comercialização e logística de transportes nos fluxos do setor agroindustrial, considerando as exportações do setor de soja in natura apenas entre a região Centro-Oeste e os mercados internacionais entre 1997 e 2015.

Portanto, não se identificou na literatura estudos de revisão sistemática da literatura internacional e que tratassem apenas dos problemas e consequências do transporte da soja oriundas de todo território brasileiro nos últimos 20 anos.

Tais evidências demonstram a relevância deste estudo que tem como objetivo levantar as limitações e os principais problemas de transporte da soja em todo o Brasil em estudos realizados entre os anos 2000 e 2019 e indexados nas principais bases de dados internacionais, destacando também suas principais consequências.

Ao sintetizar e analisar a literatura existente sobre essa temática, entende-se que os achados deste trabalho podem direcionar agendas de pesquisa no campo dos agronegócios, especialmente, no tocante à logística e o transporte de soja. Por outro lado, permite que os gestores inseridos nesta cadeia tenham conhecimento sistemático de problemas e limitações para melhorar seus processos decisórios.

\section{O TRANSPORTE E SUA IMPORTÂNCIA}

No âmbito do agronegócio, mais especificamente no setor agrícola, pode-se dizer que um sistema logístico adequado envolve, sobretudo, uma matriz de transporte eficiente e eficaz, além de outros componentes como distribuição e armazenagem dos produtos (NOVAES, 2015), sendo que esses são considerados de muita importância e são identificáveis em várias definições de logística elaboradas ao longo dos anos, tal como na proposta por Christopher (2011), que considera a logística, basicamente, como o processo de gestão estratégica na aquisição, transporte e movimentação e armazenagem de materiais.

Para Ballou (2006), o transporte representa a maior parcela dos custos logísticos de grande parte das empresas, sendo que as suas principais funções se relacionam, basicamente, com duas dimensões especificas: tempo de deslocamento e utilidade locacional (NAZÁRIO, 200o). Por isso, o transporte tem recebido grande atenção dos gestores, especialmente pela representatividade dos seus custos no valor do produto final (BATALHA; COELHO, 2011).

Assim, questões relacionadas ao transporte e seus efeitos na competitividade de um determinado produto vêm ganhando cada vez mais notoriedade em pautas dos principais debates sobre cadeias de suprimentos agroalimentares, em especial sobre cadeias como a de soja e do milho (GABAN et. al., 2017). Souza et. al. (2016), alinhados com as ideias de Petrov e Turygin (2011), entendem que o crescimento e desenvolvimento do Brasil pode ser comprometido pelo problemático sistema de escoamento da sua produção agrícola.

Considera-se, então, que o transporte no processo de escoamento da produção nacional da soja, por exemplo, é essencial para proporcionar a manutenção ou o aumento do desempenho do país frente os demais produtores, bem como para fortalecer a cadeia global desta commodity. 
O transporte, além de ser uma das três atividades primárias da logística (BALLOU, 1993), é considerado uma das principais funções logísticas (CAIXETA FILHO, 2010), fundamental para o atendimento dos objetivos logísticos que estão relacionados fortemente com a satisfação dos clientes e a obtenção de vantagens competitivas, tais como custos reduzidos e entrega de produtos conforme o momento e lugar (MENTZER et. al., 2001, NOVAES, 2015).

Diante disso, Novais (2015) define o transporte, basicamente, como o processo de deslocamento de matérias-primas e produtos acabados entre locais distintos, do ponto de vista geográfico, envolvendo uma origem e um destino intermediário ou final.

Então, considera-se que num sistema de transporte exista pelo menos três fatores fundamentais para o seu desempenho: i) custo inerentes as operações ou atividades; ii) velocidade ou tempo de movimentação; e iii) consistência ou confiabilidade do transporte no que diz respeito a variação do tempo de deslocamento (BOWERSOX et. al., 2014).

Os autores ainda salientam que o transporte está constantemente sobre influência de fatores como mão-de-obra do motorista, operação do veículo, capital investido em equipamentos e administração dos diferentes modais e estruturas de apoio, assim como perda e danos de produtos ocorridas por mal conservação de vias, os quais podem representar custos expressivos, demandando, de acordo com Scramim e Batalha (1999), ferramentas gerenciais especificas, tais como sistemas de gestão de custos e ferramentas específicas de otimização.

\section{MATERIAIS E MÉTODOS}

Conforme o que preconiza Silva e Menezes (2001), o presente estudo é caracterizado como exploratório, descritivo e qualitativo. Quanto ao procedimento técnico, utiliza-se de um Revisão Sistemática da Literatura (RSL) seguindo o protocolo de Cronin et. al. (2008).

A RSL realizada está voltada à exploração dos principais problemas relacionados ao transporte da soja brasileira, destacando suas principais consequências e os meios de solução evidenciados em trabalhos acadêmicos realizados entre os anos de 2000 e 2019. Vale destacar que a RSL se enquadra na perspectiva de revisões de tópicos que possuem um conhecimento acumulado, o qual requer uma análise e síntese dos dados (WEBSTER; WATSON, 2002).

Assim, no que se refere à análise dos dados resultantes, a pesquisa qualitativa necessita de técnicas específicas, e dentre as existentes, neste artigo a técnica de análise de conteúdo empregada é a preconizada por Bardin (1977). Sendo que o emprego da técnica de análise de conteúdo precisa seguir algumas etapas e recomendações para proceder a análise do material de forma assertiva (SILVA; FOSSÁ, 2013). Bardin (1977), portanto, apresenta três diferentes fases para a análise de conteúdo: (i) préanálise; (ii) exploração do material; e (iii) tratamento dos resultados, inferência e interpretação.

Diferentemente da revisão narrativa ou tradicional da literatura, trabalhos que utilizam essa técnica de RSL buscam delimitar, selecionar e analisar as fontes num período de tempo determinado através de uma abordagem bem definida por critérios bem estabelecidos seguindo um determinado protocolo (CRONIN et al., 2008). Assim, essa técnica pode ser utilizada para identificar o estado da arte de um determinado tema, a evolução ao longo dos anos de questões específicas de um determinado setor, assim como a relação de conteúdo entre diferentes estudos buscando propor estudos 
futuros ou até um arcabouço conceitual (GUARNIERI et. al., 2015; FILIPPI et. al., 2017; SANTOS et. al., 2018).

Portanto, de acordo com o protocolo de Cronin et. al. (2008) utilizado, as seguintes etapas devem ser seguidas rigorosamente para garantir a confiabilidade e validade do método, ou seja: (i) definição da questão de pesquisa; (ii) estabelecimento de critérios de inclusão e exclusão; (iii) seleção e acesso da literatura; (iv) avaliação da qualidade da literatura incluída na revisão; (v) analisar, sintetizar e divulgar os resultados.

Dessa forma, as etapas do protocolo utilizado são apresentadas em detalhe:

i - Definição da questão de pesquisa: Quais são as principais limitações no transporte da soja brasileira, as suas consequências e os meios de solução destacados na literatura?

ii - Estabelecimento de critérios de inclusão e exclusão: Para pesquisa inicial, foram definidas as palavras-chave com base na questão de pesquisa: transport, logistic, soy* e Brazil, palavras em inglês referentes a transporte, logística soja e Brasil, respectivamente. $\mathrm{O}$ asterisco $\left(^{*}\right)$ utilizado foi para detectar variações da palavra soy, como por exemplo soybean.

As bases de dados utilizadas foram: a) Web of Science; e, b) Scopus, por serem bases de dados que abrangem uma grande quantidade de trabalhos. O período de publicação foi restrito aos últimos 20 anos (2000 a 2019). No que se refere aos tipos de documentos publicados nesse período, definiu-se por somente artigos, desconsiderando, portanto, documentos referentes a revisão, resumos e livros. Destaca-se que os operadores booleanos OR e AND foram utilizados.

Assim sendo, a pesquisa avançada na base Web of Science foi realizada da seguinte forma: TS = ((transport OR logistic) AND soy* AND Brazil). Já na base Scopus, a pesquisa avançada realizada foi a seguinte: TITLE-ABS-KEY ((transport OR logistic) AND soy* AND Brazil). Ressalta-se que TS e TITLE-ABS-KEY são mecanismos de busca da própria base de dados Web of Science e Scopus, respectivamente, e que proporciona o retorno de trabalhos que tenham as palavras definidas e combinadas com os operadores booleanos apenas no Título, no Resumo e nas Palavras-chave de cada artigo, isto é, pode ser que no corpo do trabalho tenha essas palavras, mas com esses mecanismos específicos utilizados elas não são consideradas, não resultando trabalhos muito genéricos que dificultam a seleção.

iii - Seleção e acesso da literatura: Aplicados os princípios supracitados e considerando a incidência das palavras-chaves apenas no título, resumo e palavraschave dos artigos em cada base de dados, foram identificados 77 artigos da base Web of Science e 98 da base Scopus, sendo que 49 artigos dessa última coincidiram com a da base anterior e foram desconsiderados, restando 126 textos para avaliação.

iv - Avaliação da qualidade da literatura incluída na revisão: Dos 77 artigos da base $W e b$ of Science, 12 foram selecionados para análise, sendo que da base Scopus constatou-se que apenas 9 se encaixam no tema da pesquisa. Por fim, foram selecionados um total de 21 artigos para compor o portfólio final a ser analisado.

Destacar-se que os artigos que não se adequaram ao tema tinham as seguintes características: sem aderência das palavras-chave; objeto de estudo não era o Brasil; pesquisas voltadas à sistemas produtivos de biocombustível e biomassa, adubação do solo, uso de pesticidas; tratativas sobre outros produtos agrícolas; questões sobre o uso 
da terra; artigos de revisão; foco em métodos de cálculo de índice de preço; abordagem de outras atividades da logística que não era o transporte.

v - Análise dos artigos: Para essa análise, foi elaborado um quadro comparativo com os principais problemas logísticos discutidos e relacionados às suas principais consequências e possíveis soluções destacadas pelos autores.

A Figura 1 sintetiza os procedimentos da RSL com base nas etapas descritas.

Figura 1 - Procedimentos adotados na RSL ${ }^{*}$

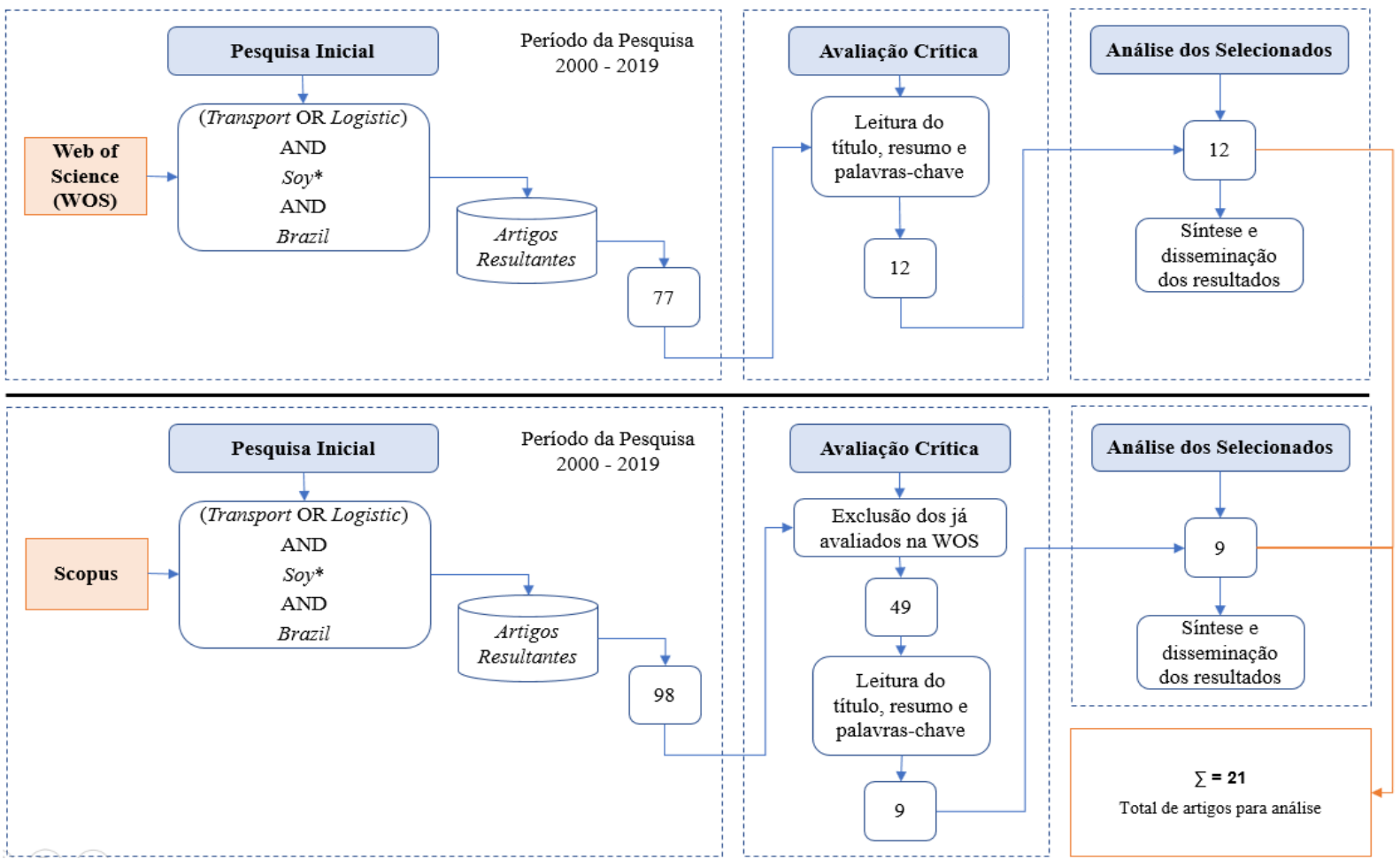

* A figura exemplifica os procedimentos em cada base de dados, Web of Science (WOS) e Scopus.

A seguir, são apresentados os resultados da pesquisa e a sua análise e discussão.

\section{RESULTADOS E DISCUSSÃO}

Inicialmente, importante destacar que fizeram parte do estudo artigos selecionados de quinze periódicos diferentes, dos quais apenas cinco tiveram mais de um estudo selecionado para análise.

Dentre eles se destaca o periódico Custos e @gronegócios Online com 3 trabalhos (LOPES et. al., 2016; LOPES; LIMA, 2017; SELEME et. al., 2017), seguido pelos periódicos com 2 trabalhos selecionados: Engenharia Agrícola (OJIMA; YAMAKAMI, 2006; BIAGGIONI; BOVOLENTA, 2010); Pesquisa Operacional (CRUZ et. al., 2010; DUBKE; PIZZOLATO, 2011); Revista em Agronegócio e Meio Ambiente (SILVA; MENEZES, 2008; SOUZA et. al., 2016) e Revista de Economia e Sociologia Rural (CORREA; RAMOS, 2010; ALMEIDA et. al., 2013), sendo que os demais periódicos tiveram somente 1 trabalho selecionado em cada: Ambio (FEARNSIDE, 2015); Benchmarking: An International Journal (MELO et. al., 2018); Brazilian 
Journal of Operations \& Production Management (PÉRA et. al., 2019); Espacios (DIONISIO et. al., 2015); Independent Journal of Management \& Production (TOLOI et. al., 2016); OLC - Oilseeds and Fats, Crops and Lipids (BERTRAND et. al., 2001); Production (ALMEIDA et. al., 2016); Research in Transportation Business \& Management (LOPES et. al., 2017); Semina: Ciências Agrárias (TELLES et. al., 2009); Transportation Research Record (FRIEND; LIMA, 2011). Esse resultado evidencia a noção de interdisciplinaridade presente em relação ao tema.

Em relação ao ano de publicação sobre o tema de pesquisa no período delimitado, na primeira década não houve resultados de trabalhos publicados na maioria dos anos, sendo que um dos principais motivos é a maior importância dada ao tema nos anos mais recentes, visto que 80\% dos trabalhos foram publicados na segunda década, como pode ser visto na Figura 2.

Figura 2 - Número de publicações por ano

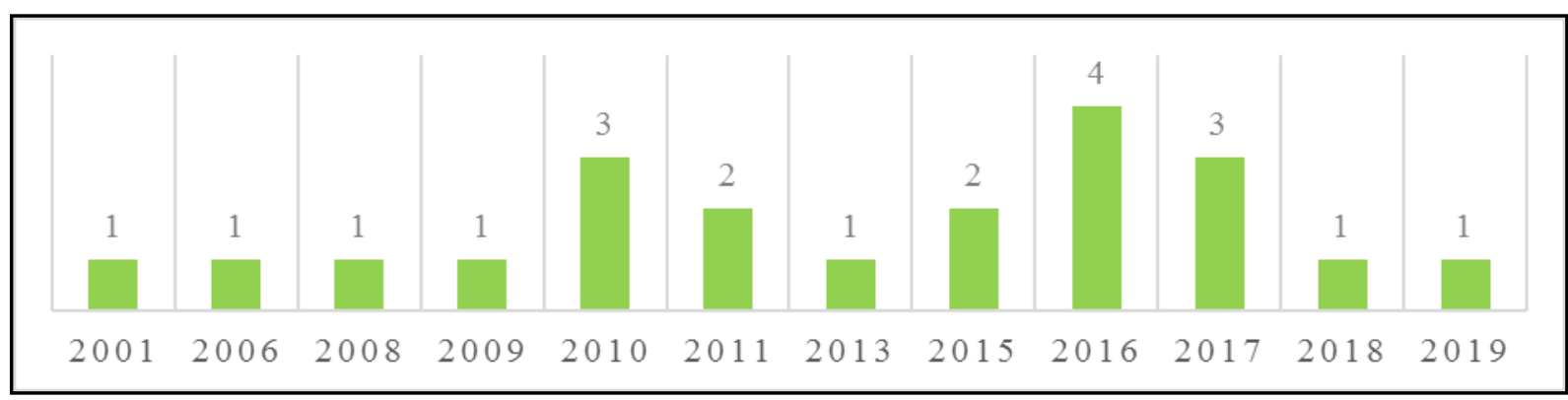

A maioria dos artigos recuperados foram publicados entre os anos de 2010 e 2017, correspondendo a mais de 70\% do total de trabalhos analisados, reforçando a consideração de importância dada às questões relacionadas ao transporte da soja no Brasil nesse período. Alguns dos motivos estão relacionados com a maior intensificação produtiva, especialmente na região Centro-Oeste, e também na região Sul do Brasil, além do aumento de cultivo da soja na região do MATOPIBA ${ }^{1}$, uma fronteira agrícola recente frente as demais. Também é importante considerar os programas e planos governamentais lançados neste período, tais como o Programa de Aceleração do Crescimento (PAC), que entrou em sua segunda fase em 2011 após ser lançado em 2007 (BRASIL, 2020), o Plano Nacional de Logística e Transporte (PNLT), criado em 2007 e revisado em 2009 e 2011 (SELEME, et. al., 2017), o Programa de Construção e Ampliação de Armazéns (PCA), criado em 2012, e o Programa de Parcerias de Investimento (PPI) de 2016 (GARCIA; VIEIRA FILHO, 2019), colocando ainda mais o setor em evidência e induzindo a realização de estudos e debates nesta área.

Com o Centro-Oeste toma posição de destaque, vale salientar que a maior parte dos trabalhos publicados entre 2010 e 2017 foram realizados nos Estados do Mato Grosso (MT), Goiás (GO) e Mato Grosso do Sul (MS), representando cerca de $72 \%$ do total de 32 estudos realizados nesses Estados entre 2000 e 2019, o que pode ser observado na Figura 3 que apresenta a relação de trabalhos por Estado.

Figura 3 - Quantidade de artigos realizados por estado entre 2000 - 2019

\footnotetext{
${ }^{1}$ Região comporta por 31 microrregiões localizadas no Maranhão, Tocantins, Piauí e Bahia. Para saber mais, acesse: <https://www.jusbrasil.com.br/diarios/104178828/dou-secao-1-13-11-2015-pg-8> .
}

Informe GEPEC, ISSN:1679-415X, Toledo, V. 25, n.1, p.261-283, jan./jun. 2021. 


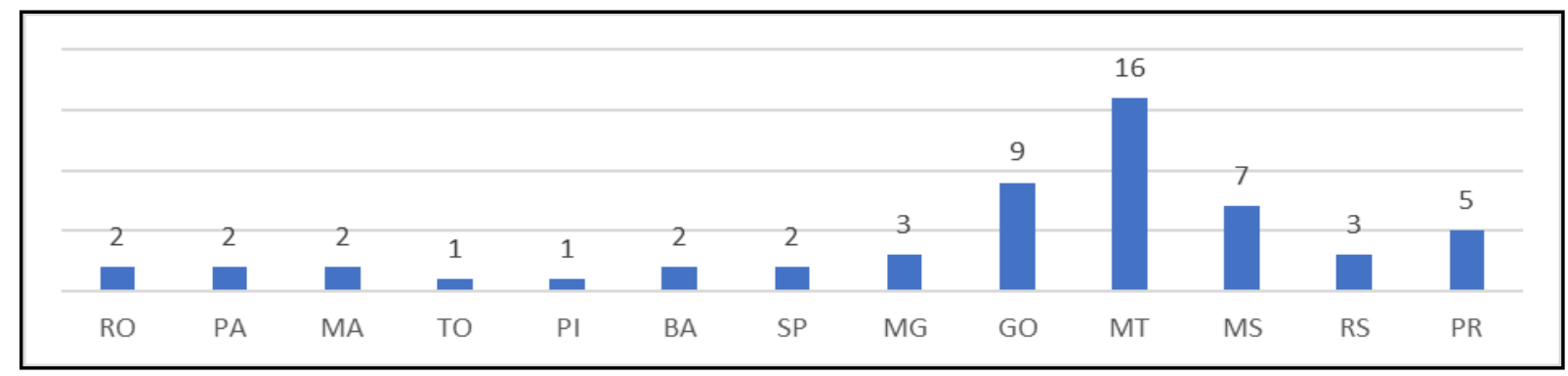

Dentre os Estados com trabalhos publicados nesses 20 anos, o MT se destaca entre os demais, com o TO e o PI ocupando o outro estremo.

Quanto aos procedimentos técnicos utilizados nos trabalhos, vale destacar que a maior parte dos trabalhos foram feitos com modelagem computacional e abordagem quantitativa, 8 no total (OJIMA; YAMAKAMI, 2006; CRUZ et. al., 2010; ALMEIDA et. al., 2013; ALMEIDA et. al., 2016; LOPES et. al., 2016; LOPES et. al., 2017; MELO et. al., 2018; PÉRA et. al., 2019), os quais utilizaram modelagem por meio do General Algebraic Modeling System (GAMS), Solver (Excel), Software de Otimização CPLEX, Análise Envoltório de Dados (DEA) e Simulação de Eventos Discretos (DES), 7 foram estudos de caso (CORREA; RAMOS, 2010; BIAGGIONI; BOVOLENTA, 2010; DUBKE; PIZZOLATO, 2011; FEARNSIDE, 2015; SOUZA et. al., 2016; TOLOI et. al., 2016; LOPES; LIMA, 2017), e 6 adotaram pesquisas do tipo survey (BERTRAND et. al., 2001; SILVA; MENEZES, 2008; TELLES et. al., 2009; FRIEND; LIMA, 2011; DIONISIO et. al., 2015; SELEME et. al., 2017).

Os com abordagem qualitativa foram 2 artigos (DIONISIO et. al., 2015; SELEME et. al., 2017), sendo que os demais são de abordagens mistas (qualitativa e quantitativa).

Os achados indicam que os custos de transporte vêm sendo considerados a principal desvantagem do setor produtivo da soja no Brasil, principalmente nas últimas décadas (BERTRAND et. al., 2001; OJIMA; YAMAKAMI, 2006; TELLES et. al., 2009; DUBKE; PIZZOLATO, 2011; SOUZA et. al., 2016; PÉRA et. al., 2019). Sendo assim, este fato apenas reflete os diversos problemas que o transporte da soja brasileira pode estar enfrentando, dificultando o bom desempenho do país no mercado global (LOPES et. al., 2017; MELO et. al., 2018).

Tais problemas estão relacionados às dimensões ou categorias diferentes, envolvendo questões distintas, mas que podem se relacionar em algum momento, tais como estruturas de apoio ao transporte, nível das estradas brasileiras, infraestrutura hidroviária, infraestrutura e equipamentos ferroviários, veículos de transporte rodoviário e fiscalização.

No Quadro 1 são apresentadas essas categorias - em ordem decrescente de quantidade de trabalhos analisados em relação a uma categoria de problema - bem como os seus respectivos problemas e os respectivos autores.

Quadro 1 - Principais problemas relacionados ao transporte da soja

\begin{tabular}{|c|c|c|}
\hline Categorias & Problemas & Autores \\
\hline
\end{tabular}




\begin{tabular}{|c|c|c|}
\hline Estrutura de Apoio & $\begin{array}{l}\text { Falta de armazéns e terminais } \\
\text { intermodais para uso de modais } \\
\text { alternativos }\end{array}$ & $\begin{array}{l}\text { Ojima e Yamakami (2006); } \\
\text { Silva e Menezes (2008); Telles } \\
\text { et. al. (2009); Friend e Lima } \\
\text { (2011); Dubke e Pizzolato } \\
\text { (2011); Almeida et. al. (2013); } \\
\text { Dionisio et. al. (2015); } \\
\text { Almeida et. al. (2016); Seleme } \\
\text { et. al. (2017); Melo et. al. } \\
\text { (2018); Péra et. al. (2019). }\end{array}$ \\
\hline Nível das estradas & $\begin{array}{l}\text { Malha rodoviária precária (asfaltos } \\
\text { degradados e falta de sinalização) }\end{array}$ & $\begin{array}{l}\text { Silva e Menezes (2008); } \\
\text { Correa e Ramos (2010); Cruz } \\
\text { et. al. (2010); Dubke e } \\
\text { Pizzolato (2011); Almeida et. } \\
\text { al. (2013); Souza et. al. (2016); } \\
\text { Lopes et. al. (2017); Seleme et. } \\
\text { al. (2017). }\end{array}$ \\
\hline $\begin{array}{l}\text { Infraestrutura } \\
\text { hidroviária }\end{array}$ & $\begin{array}{l}\text { Hidrovia com trechos inavegáveis e } \\
\text { sem acesso direto aos portos }\end{array}$ & $\begin{array}{l}\text { Telles et. al. (2009); Correa e } \\
\text { Ramos (2010); Friend e Lima } \\
\text { (2011); Lopes et. al. (2016); } \\
\text { Toloi et. al. (2016); Lopes e } \\
\text { Lima (2017); Seleme et. al. } \\
\text { (2017); Melo et. al. (2018). }\end{array}$ \\
\hline $\begin{array}{l}\text { Veículos de transporte } \\
\text { rodoviário }\end{array}$ & $\begin{array}{l}\text { Baixa capacidade de carga dos } \\
\text { caminhões e um alto consumo de } \\
\text { energia (elétrica, fóssil e etc.) }\end{array}$ & $\begin{array}{l}\text { Biaggioni e Bovolenta (2010); } \\
\text { Toloi et. al. (2016); Melo et. al. } \\
\text { (2018). }\end{array}$ \\
\hline $\begin{array}{l}\text { Infraestrutura e } \\
\text { equipamentos } \\
\text { ferroviários }\end{array}$ & $\begin{array}{l}\text { Bitolas diferentes e extensão } \\
\text { insuficiente de malha ferroviária }\end{array}$ & $\begin{array}{l}\text { Telles et. al. (2009); Dubke e } \\
\text { Pizzolato (2011); Friend e } \\
\text { Lima (2011). }\end{array}$ \\
\hline $\begin{array}{l}\text { Veículos de transporte } \\
\text { rodoviário }\end{array}$ & $\begin{array}{l}\text { Caminhões inadequados ou com idade } \\
\text { avançada }\end{array}$ & $\begin{array}{l}\text { Cruz et. al. (2010); Dubke e } \\
\text { Pizzolato (2011); Toloi et. al. } \\
\text { (2016). }\end{array}$ \\
\hline Fiscalização & Sistema de balança deficiente & $\begin{array}{l}\text { Telles et. al. (2009); Toloi et. } \\
\text { al. (2016). }\end{array}$ \\
\hline Nível das estradas & Excesso de pedágios nas estradas & Almeida et. al. (2013). \\
\hline $\begin{array}{l}\text { Infraestrutura e } \\
\text { equipamentos } \\
\text { ferroviários }\end{array}$ & $\begin{array}{l}\text { Pequena oferta de equipamentos } \\
\text { ferroviário rodantes e passagens de } \\
\text { nível em perímetros urbanos }\end{array}$ & Telles et. al. (2009). \\
\hline
\end{tabular}

Entre os problemas mais relevantes elencados no Quadro 1 e considerando as informações e resultados identificados nos artigos analisados, vale destacar interpretações importantes dessas categorias de problemas levantados de forma separada e logo após ressaltar pontos relevantes discutidos:

\section{Estruturas de Apoio}

Os problemas envolvendo as estruturas de apoio relacionam-se com a falta de armazéns e terminais intermodais. Acrescenta-se a isso as limitações sobre a decisão 
sobre o melhor momento de escoar a produção e os meios de transporte mais adequados para a soja. Isso é explicado pelo fato de ter sido identificado na literatura que uma plena adequação do sistema de transporte não depende apenas das malhas viárias disponíveis ou em condições de uso, visto que rotas sem essas estruturas foram consideradas menos eficientes em alguns estudos (OJIMA; YAMAKAMI, 2006; TELLES et. al., 2009; DUBKE; PIZZOLATO, 2011; MELO et. al., 2018).

Somada a isso, de acordo com Friend e Lima (2011); Almeida et. al. (2013); Dionísio et. al. (2015); Almeida et. al. (2016); Seleme et. al. (2017); Melo et. al. (2018) e Péra et. al. (2019), tem-se as grandes distâncias que a soja tem percorre no escoamento, acarretando numa grande dependência do setor ao modal rodoviário.

Torna-se importante evidenciar ainda que, para Bertrand et. al. (2001); Silva e Menezes (2008); Dionisio et. al. (2015) e Seleme et. al. (2017), o aumento considerável do valor do frete, as perdas quantitativa e qualitativa do produto e os veículos com funcionalidade de "armazéns móveis" também acabam sendo consequências significativas dessas limitações.

Dessa forma, os produtores e transportadores são colocados frente a um grande desafio, o qual é importante ser superado para que se consiga melhores margens e que se faça jus ao alcance de uma maior produção e produtividade (DIONISIO et. al., 2015; ALMEIDA et. al., 2016).

Nesse sentido, de acordo com Silva e Menezes (2008); Friend e Lima (2011); Almeida et. al. (2013); Seleme et. al., (2017); e Péra et. al., (2019), a disponibilidade dessas estruturas de apoio com a capacidade necessária pode favorecer o uso dos modais alternativos, ferroviário e hidroviário, proporcionando uma significativa redução dos custos de escoamento da soja, por apresentarem maior eficiência enérgica e maior capacidade de carga. Assim, é notório a importância da ampliação ou instalação de mais terminais intermodais, ferroviários e portos. Para tanto, os autores Dionisio et. al. (2015) e Seleme et. al. (2017) destacam que a conclusão do Plano Nacional de Logística e Transporte (PNLT) e do Plano de Aceleração do Crescimento (PAC), propostos pelo Estado brasileiro nos respectivos momentos governamentais, seria uma forma de conseguir essas melhorias. Acrescenta-se o aumento das Parcerias Público-Privadas, o que proporcionaria mais recurso para os investimentos estruturais.

Contudo, embora as rotas com estruturas de apoio tendam a ser mais eficientes, principalmente se tratando dos terminais intermodais, há de se considerar que pode existir um limite para a intermodalidade ou multimodalidade, visto que Melo et. al. (2018) identificam em seu estudo de modelagem que as rotas mais eficientes no Brasil utilizaram dois modais, já as com três ou mais modais induziram a ineficiência do transporte da soja. Souza et. al. (2016) concordam e acrescentam que isso ainda pode aumentar o tempo em que o produto fica em trânsito estocado;

\section{Nível das Estradas}

O nível das estradas relaciona-se com a malha rodoviária precária e ao excesso de pedágios nas estradas. Para Cruz et. al. (2010); Dubke e Pizzolato (2011); Lopes et. al. (2017) e Seleme et. al. (2017), grande parte das rodovias do país, especialmente as mais importantes para escoamento das safras, como a BR 163 e BR 364, vem apresentando trechos despavimentados ou com asfaltos degradados. No entanto, as vias que são pavimentadas muitas vezes apresentam excesso de pedágio, além da falta de acostamento e sinalização (CORREA; RAMOS, 2010; ALMEIDA et. al., 2013). 
Nesse contexto, a principal consequência desses problemas se encontra no aumento dos custos operacionais, de manutenção e com combustível, elevando os valores do frete de forma considerável e recorrente. Alia-se à limitada opção de rotas ou modais que apresentam custos mais baixos (SILVA; MENEZES, 2008; SOUZA et. al., 2016; SELEME et. al., 2017).

Os autores Ojima e Yamakami (2006); Silva e Menezes (2008); Cruz et. al. (2010); Correa e Ramos (2010); Lopes et. al. (2017) e Seleme et. al. (2017) propõem as Parcerias Público-Privadas como um meio de solução promissor desses problemas relacionados à investimentos na melhoria das estradas brasileiras de escoamento da soja, principalmente, no que tange necessidade de manutenção e de realização de pavimentação, duplicações ou construções de terceira faixa nas rodovias. Reclama ainda da falta de benefícios e maior agilidade na aprovação e liberação dos projetos desenvolvidos e contratos justos para ambas as partes, o que geralmente envolve agências nacionais e a esfera legislativa do Estado.

Vale destacar que, além das limitações citadas anteriormente, o transporte rodoviário tende a ser o modal mais preferido por apresentar vantagens como uma maior flexibilidade, rapidez e acesso mais fácil às áreas específicas que não são alcançadas por outros modais (SILVA; MENEZES, 2008; CORREA, RAMOS, 2010; SOUZA et. al., 2016; SELEME et. al., 2017).

Embora não seja possível afirmar que os problemas identificados até 2013 ainda persistem, contudo nota-se que até recentemente foram sugeridos significativos investimentos nessa área, como por exemplo, pelos autores Lopes et. al. (2017);

\section{Infraestrutura Hidroviária}

No que diz respeito aos problemas envolvendo infraestruturas hidroviárias, Telles et. al. (2009); Correa e Ramos (2010); Friend e Lima (2011); Melo et. al. (2018) destacaram a extensão viária sem acesso direto aos portos e, principalmente, a existência de trechos de alguns rios importantes do país que apresentam partes estreitas e sem a profundidade necessária. Isso corroboram com Lopes et. al. (2016) e Toloi et. al. (2016) que levantaram que a inviabilidade de navegação ainda é observada em vários trechos de hidrovias como a Tocantins-Araguaia e Tietê-Paraná.

Dessa forma, Toloi et. al. (2016) e Lopes e Lima (2017) salientam que sem acesso direto aos portos, torna-se necessário paradas para transbordo, o que geralmente é caro e eleva os custos de transporte de forma significativa, além de proporcionar perdas de quantidade e de qualidade dos produtos transportados.

Por sua vez, Friend e Lima (2011) e Melo et. al. (2018) destacam que tais condições das hidrovias brasileiras acabam não possibilitando a combinação de mais de três barcaças e um reboque, favorecendo a baixa eficiência do transporte, haja visto que as vias navegáveis tendem a ser mais eficientes quando apenas com grandes combinações de barcaças. Pode-se tomar como exemplo um dos principais concorrentes do Brasil em termos de produção de soja, os EUA, considerado um país com transporte eficiente e que utiliza o rio Mississippi como principal rota de transporte hidroviário, destaca-se Melo et. al., 2018, quando acrescenta que nessa hidrovia é possível navegar com uma combinação de 15 barcaças e um reboque.

Nessa perspectiva, Toloi et. al. (2016) ainda destacam que a capacidade de um rebocador de barcaças de puxar ou empurrar até 1.500 toneladas (ton) equivale a 35 caminhões do tipo Bi-trem que possuem cada um a capacidade de 43 t. de carga útil, considerado por Melo et. al. (2018) como um dos principais veículos no transporte da 
soja brasileira. Pode-se considerar que uma das vantagens do modal hidroviário é a grande capacidade de carga e a eficiência no transporte de longas distâncias.

Desta maneira, considerando tais problemas e as vantagens desse modal, alguns autores levantam possíveis meios de resolvê-los. Dubke e Pizzolato (2011); Fearnside (2015); Lopes e Lima (2017); Melo et. al. (2018) destacam a construção de eclusas e/ou dragagem dos rios para comportar embarcações com calado maior ${ }^{2}$; já Telles et. al. (2009) e Seleme et. al. (2017) destacam as Parcerias Público-Privadas; Correa e Ramos (2010) levantam a necessidade de desenvolver a cabotagem no país; enquanto Toloi et. al. (2016) salientaram a necessidade de redução do fluxo de água das usinas hidrelétricas, a fim de elevar o nível da água;

\section{Infraestrutura Ferroviária}

Em relação às questões de infraestrutura das vias férreas, Telles et. al. (2009), corroborados por Dubke e Pizzolato (2011) e Friend e Lima (2011), destacam as bitolas diferentes das linhas férreas e a curta extensão da malha ferroviária, além da pouca disponibilidade de equipamentos ferroviários rodantes e de qualidade aceitável, bem como as passagens de nível em perímetros urbanos como principais problemas.

Sobre as bitolas diferentes e a reduzida malha ferroviária disponível, as principais consequências evidenciadas foram a impossibilidade de uma maior utilização do modal ferroviário. Já sobre os equipamentos ferroviários rodantes, destaca-se que os trens operam constantemente com a capacidade abaixo do ideal e, por fim, sobre as passagens de nível em áreas urbanas, além do aumento do risco de acidentes envolvendo pedestres ou automóveis, ainda é possível salientar que a velocidade média dos trens reduz cerca de $87,5 \%$ da sua velocidade ideal de $40 \mathrm{Km} / \mathrm{h}$ (TELLES et. al., 2009), reduzindo a eficiência operacional do modal em questão.

Por conseguinte, os autores Telles et. al. (2009) e Friend e Lima (2011) enfatizam que um possível meio para solucionar esses problemas começa pela iniciativa do Estado, que deve estudar a possibilidade de atribuir à iniciativa privada a responsabilidade pela execução e manutenção de parte dos investimentos estruturais dessa magnitude e buscar o estabelecimento de Parcerias Público-Privadas.

Dessa forma, assim como é notório os poucos trabalhos que ditam sobre problemas relacionados à infraestrutura ferroviária, no que tange o tema deste trabalho, também é evidente que não se pode afirmar se tais problemas são recorrentes nos anos atuais e serão nos subsequentes. No entanto, o uso do modal ferroviário ainda é fortemente recomendado pelos autores Dionisio et. al. (2015) e Melo et. al. (2018), os quais afirmam que esse modal pode tornar o escoamento da soja mais eficiente, visto a sua grande capacidade de transporte de carga, principalmente aquelas de baixo valor agregado e que percorrem maiores distâncias, como é o caso da soja oriunda do CentroOeste, maior região produtora do Brasil;

\section{Veículos de Transporte Rodoviário}

Considerando que o aumento da produção e da produtividade de soja podem esbarrar na capacidade de escoamento do País, os autores Biaggioni e Bovolenta (2010)

${ }^{2} \mathrm{O}$ calado corresponde à altura do espaço ocupado pelas embarcações dentro da água. 
e Toloi et. al. (2016) destacam como problema a baixa capacidade de carga dos caminhões, o que, segundo Melo et. al. (2018), faz surgir a necessidade de realizar uma grande quantidade de viagens para o escoamento da safra, acarretando em congestionamentos nas rodovias e, ainda, favorecendo o aumento do índice de acidentes nas estradas.

Entretanto, também é identificado o problema do alto consumo de energia dos veículos de transporte rodoviário, seja de fonte elétrica, fóssil ou biológica (mão de obra), induzindo a uma significativa ineficiência energética do transporte da soja, principalmente quando comparados com outros modais (BIAGGIONI; BOVOLENTA, 2010; TOLOI et. al., 2016; MELO et. al., 2018).

Não obstante, Cruz et. al. (2010); Dubke e Pizzolato (2011) e Toloi et. al. (2016) também colocam em evidência os caminhões inadequados e com idade avançada, os quais favorecem a grandes perdas do produto em termos de quantidade e qualidade, além de consumir maiores quantidades de combustíveis, pneus, óleos lubrificantes e etc, elevando os custos de transporte da soja e induzindo à ineficiência do modal.

Assim, torna-se necessário mais investimentos, seja do poder público ou por meios das Parcerias Público-Privadas, para que seja possível contornar esses problemas (OJIMA; YAMAKAMI, 2006, SELEME et. al., 2017), proporcionando uma maior utilização da intermodalidade com o modal ferroviário e o hidroviário, e priorizando o modal rodoviário em distancias mais curtas (BIAGGIONI; BOVOLENTA, 2010; ALMEIDA et. al., 2013; MELO et. al., 2018);

\section{Fiscalização}

Atrelado a necessidade de realização de mais viagens, devido à baixa capacidade dos veículos rodoviários e a ineficiência energética desses, tem-se observado a deficiência do sistema de balanças nas estradas brasileiras, visto que esse problema, consequentemente, acaba favorecendo o tráfego desses veículos com o excesso de peso (sobrecarga), o que reduz, assim, a vida útil da pavimentação das estradas (TELLES et. al., 2009; TOLOI et. al., 2016).

Nessa perspectiva, Toloi et. al., (2016) ainda ressaltam consequências adicionais, tais como as restrições ao tráfego rodoviário, os impasses que ocorrem nas cidades, maiores avarias e necessidade de manutenção causada pelo desgaste dos componentes dos veículos e as adaptações (usando madeira no corpo do caminhão) para comportar maior volume de carga.

Então, a reestruturação dos órgãos de fiscalização do governo pode ser uma alternativa na abordagem dos problemas acima descritos (TELLES et. al., 2009), visto que até então o poder público é o responsável por funções importantes como essa.

Diante desses problemas categorizados e discutidos, é notório uma matriz de transporte bastante fragilizada que reduz de forma considerável a competitividade global do país frente aos seus principais concorrentes, os EUA e a Argentina, o que está alinhado com os dados de Alvarenga e Lobo (2020), sobretudo quando comparado a países como a Rússia, Alemanha, Índia e EUA, especialmente em relação ao modal mais utilizado, o rodoviário, pois o Brasil tem a menor quantidade de rodovias pavimentadas, cerca de 213 mil km, e também em relação a um dos modais que mais ajudaria na eficiência do transporte da soja se amplamente utilizado, o ferroviário, apresentando a menor extensão ferroviária com apenas $30 \mathrm{mil} \mathrm{km.}$

As evidências também ressaltam alguns pontos de atenção, o principal é a necessidade de redistribuição das cargas de soja em grãos de maneira mais eficiente entre os possíveis meios de transportes disponíveis, e embora Lopes e Lima (2017) 
digam que o setor logístico brasileiro é caracterizado pela inovação e pela busca de alternativas para o sistema de transporte, os esforços têm se mostrado pouco eficazes diante dos problemas destacados, o setor ainda deve buscar a utilização ampla de mecanismos, equipamentos e sistemas inovadores (transporte, terminais, armazenagem etc.).

Garcia e Vieira Filho (2019) ressaltam que ao longo dos anos os produtores rurais e empresas agrícolas vêm buscando contornar os efeitos negativos desses problemas através da verticalização de serviços logísticos, aplicação de investimentos em armazenagem, aprimoramento de sistemas de gestão e a busca constante por ganho de produtividade no campo, dentre outros, mas a omissão da União, estados e municípios acaba sendo um entrave gigantesco na resolução desses problemas logísticos, visto a capacidade limitada em termos técnico, institucional e financeiro dos produtores e empresas agrícola.

Assim, uma maior atenção do Estado é necessária especialmente nos problemas fora do alcance de outros agentes da cadeia, como é o caso dos serviços de fiscalização que está intimamente relacionado aos níveis das estradas e com os veículos que por ali trafegam. A abordagem de sistemas de fiscalização mais eficiente se torna indispensável, como por exemplo, com a implantação de sistemas inovadores como o de pesagem em movimento de alta velocidade (HS-WIM) capaz de apurar de forma mais eficiente o peso das cargas de soja escoadas pelas estradas brasileiras (BOCK; BRITO, 2018). Para Brito et. al. (2014), embora sistemas de balanças tradicionais ainda apresentem uma maior eficácia, o sistema HS-WIM é mais eficiente é apresenta benefícios como infraestrutura barata; operacionalização automática sem necessidade de agentes de pesagem; monitoramento contínuo com possibilidade de $100 \%$ do fluxo de veículos pesados; velocidade operacional maior; custo de operação menor; e sobretudo, garantindo aos transportadores não infratores a gratificação de não serem onerados com os gastos de tempo em balanças tradicionais.

Finalmente, associado ao custo de transporte alarmante devido as limitações logísticas que provocam grandes efeitos negativos na competitividade e produtividade dos produtos agrícolas, o setor apresenta um elevado custo de oportunidade e é onde os recursos escassos do Estado mais se dissipa, impactando diretamente em outros setores importantes e necessitados da economia brasileira, como educação e saúde.

\section{CONSIDERAÇÕES FINAIS}

Considerando a importância da soja para a economia do Brasil, haja visto sua posição de destaque como um dos maiores produtores e exportadores do mundo, o sistema de transporte precário do país é um fator que não favorece a sua competitividade no mercado global. Baseado nisso, essa pesquisa objetivou identificar os principais problemas encontrados no transporte da soja no Brasil, destacando suas principais consequências evidenciadas nos últimos 20 anos. Para tanto, utilizou-se da RSL para sintetização do conhecimento gerado a cerca desse assunto ao longo dos anos e da análise de conteúdo para análise e categorização dos achados dessa RSL.

Os resultados mostram que os principais problemas evidenciados relacionamse às estruturas de apoio, que envolve a falta de armazéns e terminais intermodais; o nível das estradas brasileiras, que envolve a malha rodoviária precária e ao excesso de pedágios nas estradas; e a infraestrutura hidroviária, que por sua vez envolve a extensão viária sem acesso direto aos portos e, principalmente, a existência de trechos de alguns rios importantes do país que apresentam pontos estreitos e partes sem a profundidade necessária. 
Por outro lado, os problemas que foram menos citados na literatura, mais que também são importantes, relacionam-se à infraestrutura e equipamentos ferroviários, que envolve as bitolas diferentes das linhas férreas existentes e a falta de malha ferroviária, a pouca disponibilidade de equipamentos ferroviário rodantes e com qualidade aceitável, bem como as passagens de nível em perímetros urbanos; os veículos de transporte rodoviário, que envolve a baixa capacidade de carga dos caminhões e ao alto consumo de energia dos veículos de transporte rodoviário e os caminhões inadequado e com idade avançada; e a fiscalização que envolve o sistema deficiente de balança nas estradas brasileiras.

Diante desses principais problemas identificados, a grande dependência do modal rodoviário pela pouca utilização dos modais hidroviários e ferroviários, as perdas quantitativas e qualitativa da soja pela maior necessidade de transbordo, baixos níveis das estradas, uso dos veículos inadequados e falta de estrutura de armazéns, bem como a elevação dos custos totais do transporte são as consequências mais evidenciadas.

Por conseguinte, nota-se um consenso entre os autores que um meio promissor na tentativa de resolver os problemas destacados neste estudo é o estabelecimento de Parcerias Público-Privadas, que além proporcionar a ampliação dos recursos para execução de projetos de infraestruturas de transporte, envolvendo os três principais modais de transporte tratados aqui, visto que obra desse tipo geralmente demandam grandes quantias, também poderá proporcionar o planejamento em conjunto das partes envolvendo tais questões e o desenvolvimento de políticas públicas mais abrangentes.

À vista dessas considerações, acredita-se que uma das principais limitações desta pesquisa está no fato de não ter sido tratado de questões ambientais e sociais relacionadas aos problemas do transporte da soja no Brasil, visto que o estudo se atentou mais para as questões econômicas. Destaca-se também, como limitações de estudo, o protocolo utilizado que apresenta etapas que excluem estudos da análise, além do uso de apenas palavras-chave em inglês na busca em ambas bases de dados.

Finalmente, buscando direcionar futuros estudos a partir dessa pesquisa, algumas questões importantes que podem ser consideradas são destacadas: a) Se existe alguma relação, qual o grau de influência desses problemas no nível de produção da soja brasileira? b) Quais as peculiaridades envolvendo as relações contratuais que podem dificultar o estabelecimento de Parcerias Público-Privado? c) Quais as principais barreiras que impedem a solução desses problemas? d) Até que ponto esses problemas podem afetar o estabelecimento de uma Gestão da Cadeia de Suprimentos eficaz? e) Quais as implicações ambientais e sociais que esses problemas identificados provocam ou podem provocar?

\section{REFERÊNCIAS}

ALMEIDA, M. S.; AMARAL, M.; MORABITO, R. Um estudo sobre localização de terminais intermodais na rede de escoamento da soja brasileira. Production, v. 26, n. 3, p. 562-580, Jun./Set., 2016. 
ALMEIDA, C. A.; SELEME, R.; NETO, J. C. Rodovia Transoceânica: uma alternativa logística para o escoamento das exportações da soja brasileira com destino à China. Revista de Economia e Sociologia Rural, Piracicaba-SP, v. 51, n. 2, p. 351-368, Abr./Jun., 2013.

ALMEIDA, T. E. Desafios na gestão logística de uma microempresa fabricante de biscoitos na perspectiva da integração das atividades primárias e de apoio.

Research, Society and Development, v. 9, n. 8, p. 1-23, 2020.

ALVARENGA, H.; LOBO, A. Perspectivas para a infraestrutura brasileira.

Especialistas em Logística e Supply Chain (ILOS), 2020. Disponível em: <https://www.ilos.com.br/web/perspectivas-para-a-infraestrutura-brasileira/>. Acesso em: o9 Dez. 2020.

BALLOU, R. H. Logística empresarial: transportes, administração de materiais e distribuição física. São Paulo: Atlas, 1993.

BALLOU, R. H. Gerenciamento da Cadeia de Suprimentos: Logística Empresarial. Bookman Editora, 2006.

BARBOZA, M. A. M. A ineficiência da infraestrutura logística do Brasil. Revista Portuária, 2014.

BARDIN, L. Análise de conteúdo. Lisboa: Edições 70, 1977.

BATALHA, M. O.; COELHO, F. B. Análise dos cenários para suprimento de defensivos agrícolas no complexo da cana-de-açúcar. Revista Eletrônica

Sistemas \& Gestão, v. 6, p 573-548, 2011.

BERTRAND, J. P.; WANIEZ, P.; BRUSTLEIN, V.; STEPE, U. Local factors in the competitiveness of the soybean sector in Brazil. OCL - Oilseeds and fats, crops and lipids, v. 8, n. 3, p 223-229, Mai./Juin., 2001.

BIAGGIONI, M. A. M.; BOVOLENTA, F. C. Balanço energético comparativo para rotas de escoamento de soja. Engenharia Agrícola, Jaboticabal, v.30, n.4, p 587599, Jul./Ago., 2010.

BOCK, A. L.; BRITO, L. A. T. Fiscalização de cargas rodoviárias: estudo comparativo entre posto de pesagem veicular móvel e um sistema wim implantado na rodovia br290/rs-freeway. In: Anais do XXXII Congresso de Pesquisa e Ensino em Transportes, p. 3550-3561, 2018.

BOWERSOX, D. J.; CLOSS, D. J.; COOPER, M. B.; BOWERSOX, J. C. Gestão logística de cadeias de suprimentos. Porto Alegre: AMGH, ed. 4, 2014.

BRITO, L.; BOCK, A. L.; CERATTI, J. A. P.; NÚÑEZ, W. P. Sistema de Pesagem em Movimento-WIM: Instalação e Calibração em Pista Experimental para Monitoramento e Classificação do Espectro de Cargas Rodantes da BR-290/RSFreeway. Revista de Engenharia Civil IMED, v. 1, n. 1, p. 29-41, 2014. 
BRASIL, Ministério do Planejamento. Programa de Aceleração do Crescimento (PAC). 2020. Disponível em: <http://pac.gov.br/sobre-o-pac>. Acesso em: 15 Dez. 2020.

CAIXETA FILHO, J. V. Logística para a agricultura brasileira. Revista Brasileira de Comércio Exterior, v. 103, p. 18-30, 2010.

CAUDURO, R. F.; LEMOS, I. S. Análise da logística reversa de pós-venda numa empresa de manutenção de aeronaves. Revista Gestão e Desenvolvimento, v. 7, n. 1, p. 31-40, 2010.

CEPEA - Centro de Estudos Avançados em Economia Aplicada. PIB do agronegócio brasileiro. 2020. Disponível em:

<https://www.cepea.esalq.usp.br/br/pib-do-agronegocio-brasileiro.aspx>. Acesso em: 03 Dez. 2020.

CHRISTOPHER, M. Logística e Gerenciamento da Cadeia de Suprimentos Tradução da $4^{\mathrm{a}}$ edição norte-americana. São Paulo: Cengage Learning, 2011.

CORREA, V. H. C.; RAMOS, P. A Precariedade do Transporte Rodoviário Brasileiro para o Escoamento da Produção de Soja do Centro-Oeste: situação e perspectivas.

Revista de Economia e Sociologia Rural. Brasília, v. 48, n.2, p. 447-472, Abr./Jun. 2010.

CRONIN, P.; RYAN, F.; COUGHLAN, M. Undertaking a Literature Review: a step-bystep approach. British Journal of Nursing, v. 17, n. 1, p. 38-43, 2008.

CRUZ, B. C. B.; PIZZOLATO, N. D.; CRUZ, A. B. D. An application of the spatial equilibrium model to soybean production in tocan-tins and neighboring states in Brazil. Pesquisa Operacional, Rio de Janeiro, v. 30, n. 2, Mai/Ago. 2010.

DASKIN, M.S. Logistics: an overview of the state of the art and perspectives on future research. Transportation Research - A, v. 19A, n. 5/6, p. 383-398, 1985.

DIONISIO, E. A.; FREITAS, W. J.; IGNÁCIO, P. S. A. Prospecção de cenários para uma cadeia agroexportadora de soja. Espacios, v. 36, n. 22, p. 1-12, 2015.

DUBKE, A. F.; PIZZOLATO, N. D. Location model of specialized terminals for soybean exports in brazil. Pesquisa Operacional, v. 31, n. 1, p. 21-40, 2011.

FILARDO, M. L. R.; ILARIO, A. A.; SILVA, G. D.; CARVALHO, M. A. A logística da exportação de soja do Estado de Mato Grosso para o Porto de Santos. Revista de Economia Mackenzie, v. 3, n. 3, 2005.

FILIPPI, A.C.G.; GUARNIERI, P. FARIAS, J.S. Logística agroindustrial: uma revisão sistemática nos anais de Congresso da Sober. Revista em Agronegócio e Meio Ambiente, v. 10, n. 4, p. 1077-1112, Out./Dez. 2017. 
FRIEND, J. D.; LIMA, R. S. Impact of Transportation Policies on Competitiveness of Bra-zilian and U.S. Soybeans: from field to port. Transportation Research

Record, v. 2238, ed. 1, p. 61-67, 2011.

FEARNSIDE, P. M. Amazon dams and waterways: Brazil's Tapajo's Basin plans. Ambio, v. 44, ed. 5, p. 426-439, 2015.

GABAN, A. C.; GUARNIERI, P. Identificação de gargalos na logística agroindustrial: revisão sistemática da literatura. In: Congresso da Sociedade Brasileira de Economia, Administração e Sociologia Rural - SOBER. Anais... João Pessoa - PB: UFPB, 2015. Disponível em: <https://www.researchgate.net/publication/317004026_IDENTIFICACAO_DE_GA RGALOS_NA_LOGISTICA_AGROINDUSTRIAL_REVISAO_SISTEMATICA_DA_L ITERATURA>. Acesso em: o8 Dez. 2020.

GABAN, A. C.; MORELLI, F.; BRISOLA, M. V.; GUARNIERI, P. Evolução da produção de grãos e armazenagem: perspectivas do agronegócio brasileiro para 2024/25. iGepec, Toledo, v. 21, n. 1, p. 28-47, Jan/Jun, 2017.

GARCIA, J. R.; VIEIRA FILHO, J. E. R. Logística e Armazenagem. In: VIEIRA FILHO, J. E. R (Org.). Diagnóstico e Desafios da Agricultura Brasileira. Rio de Janeiro: IPEA, p. 59-88, 2019.

GUARNIERI, P.; SOBREIRO, V. A.; NAGANO, M. S.; SERRANO, A. L. M. The challenge of selecting and evaluating third-party reverse logistics providers in a multicriteria perspective: A Brazilian case. Journal of Cleaner Production, v. 96, p. 209-219, 2015 .

GUDOLLE, M. F.; ZAMBERLAN, J. F.; BORTOLOTTO, R. P.; MERA, C. M. P.; PASINI, M. P. B.; SALAZAR, R. F. S. Logística da soja em grão e os custos dos modais de transporte. Desenvolvimento Agropecuário Sustentável, ed. 1, p. 61-73, 2019.

LOPES, H. S.; LIMA, R. S.; FERREIRA, F. C. A cost optimization model of transportation routes to export the Brazilian soy-bean. Custos e @gronegócio on line, v. 12, n. 4, p. 90-109, Out./Dez., 2016.

LOPES, H, S.; LIMA, R. S.; LEAL, F.; NELSON, A. C. Scenario analysis of Brazilian soybean exports via discrete event simulation applied to soybean transportation: The case of Mato Grosso State. Research in Transportation Business \& Management, v. 25, p. 66-75, 2017.

LOPES, H. S.; LIMA, R. S. Alternatives for the soybean exportation in Brazil: a cost based analysis for. Custos e @gronegócio on line, v. 13, n. 1, Jan/Mar., 2017.

MELO, I. C.; JUNIOR, P. N. A.; PERICO, A. E.; GUZMAN, M. G. S.; REBELATTO, D. A. N. Benchmarking de corredores e rotas de transporte de mercadorias com análise de envelope de dados (DEA). Benchmarking: An International Journal, v. 25, n. 2, p. 713-742, 2018. 
MENTZER, J. T.; DEWITT, W.; KEEBLER, J. S.; MIN, S.; NIX, N. W.; SMITH, C. D.; ZACHARIA, Z. G. Defining supply chain management. Journal of Business logistics, v. 22, n. 2, p. 1-25, 2001.

NAZÁRIO, P. Papel do transporte na estratégia logística. In: FLEURY, P. F; WANKE, PETER; FIGUEIREDO, K. F. (Org.). Logística empresarial: a perspectiva brasileira. São Paulo: Editora Atlas S.A, p. 125-132, 2000.

NOVAES, A. Logística e gerenciamento da cadeia de distribuição. Rio de Janeiro: Elsevier, ed. 4, 2015.

OJIMA, A. R. L. O.; YAMAKAMI, A. Quadratic programming model in the analysis of the logistical movement and marketing of brazilian soybean. Engenharia Agrícola, Jaboticabal, v. 26, n. 2, p. 552-560, Mai/Ago., 2006.

PÉRA, T. H.; BARTHOLOMEU, D. B.; SU, C. T.; CAIXETA FILHO, J. V. Evaluation of green transport corridors of brasilian soybean exports to china. Brazilian Journal of Operations \& Production Management, v. 16, n. 3, p. 398-412, 2019 .

PETROV, M.B.; TURYGIN, O.M. Increase of transport-logistic servicing efficiency of Sverdlovsk region's agroindustrial complex (on the example of grain cargoes transportation). Economy of Region, n. 4, p. 68-78, 2011.

PONTES, H. L. J.; DO CARMO, B. B. T.; PORTO, A. J. V. Problemas logísticos na exportação brasileira da soja em grão. Revista Eletrônica Sistemas \& Gestão, v. 4, n. 2, p. 155-181, 2009.

SANTOS, R. R. D.; GUARNIERI, P.; BRISOLA, M. V. Logística reversa de resíduos das atividades agrossilvipastoris e agroindustriais: uma revisão sistemática da literatura. Revista em Agronegócio e Meio Ambiente, v. 11, n. 2, p. 573-597, Abri./Dez. 2018.

SANTOS, J. D. S.; PEREIRA, L. A. G. Logística de transportes do agronegócio e exportações de soja no centro-oeste brasileiro. Revista Eletrônica do Curso de Geografia - UFG/REJ, n. 34, p. 131-154, 2019.

SCRAMIM, F. C. L.; BATALHA, M. O. Supply Chain Management Em Cadeias Agroindustriais: discussões acerca das aplicações no setor lácteo brasileiro. II Workshop Brasileiro de Gestão de Sistemas Agroalimentares PENSA/FEA/USP Ribeirão Preto, 1999.

SELEME, R.; ZATTAR, I. C.; SILVA, W. A.; SCHAKER, F. R. C.; NERY, V. F. S. O. Alternativas para melhoria de indicadores logísticos da soja brasileira com base no Plano Nacional de Logística e Transportes (PNLT). Custos e @gronegocios Online, v. 13, n. 4, p. 190-210, Out/Dez. 2017.

SILVA, E.L.; MENEZES, E.M. Metodologia da Pesquisa e Elaboração de Dissertação. 3 ed. revisada e atualizada, Florianópolis, 2001. 
SILVA, M. S.; MENEZES, T. M. Corredor de Escoamento Noroeste: alternativa logística para produtores agrícolas das regiões centro e norte do estado do mato grosso. RAMA: Revista em Agronegócio e Meio Ambiente, v. 1, n. 1, p. 37-44, Jan./Abr., 2008.

SILVA, A.H.; FOSSÁ, M.I.T. Análise de conteúdo: exemplo de aplicação da técnica para análise de dados qualitativos. In: ENCONTRO DE ENSINO E PESQUISA EM ADMINISTRAÇÃO E CONTABILIDADE, 4., 2013, Brasília. Anais... Brasília, 2013.

SOUZA, R. O.; CREMASCO, C. P; GABRIEL FILHO, L. R. A. Análise dos valores de frete da soja a granel nos sistemas unimo-dal e multimodal de transporte. Revista em Agronegócio e Meio Ambiente, v. 9, n. 4, p. 819-837, Out./Dez., 2016.

TELLES, T. S.; GUIMARÃES, M. F.; ROESSING, A. C. The transport infrastructure go-ahead the expansion of soybean cultivation in Brazil. Semina: Ciências

Agrárias, Londrina, v. 30, suplemento 1, p. 1109-1122, 2009.

TOLOI, R. C.; JUNIOR, M. F.; REIS, J. G. M.; VENDRAMETTO, O.; NETO, P. L. O. C. Droughts in the tietê-paraná waterway: impacts on the direct, indirect and hidden costs in the transportation of soybean. Independent Journal of Management \& Production, v. 7, n. 2, Apr/Jun., 2016.

USDA - United States Department of Agriculture. Oilseeds: World Markets and trade. Washington, D.C.-EUA: USDA, 2020. Disponível em:

$<$ https://usda.library.cornell.edu/concern/publications/tx31qh68h?locale=en $>$. Acesso em: 07 mar. 2020.

WEBSTER, J.; WATSON, R. T. Analyzing the Past to Prepare for the Future: Writing a Review. MIS Quarterly: Management Information Systems, v. 26, n. 2, p. 12, 2002. Disponível em: <www.jstor.org/stable/4132319>. Acesso em: o9 nov. 2019. 


\section{Sobre o(s) Autor(es):}

\section{Maicon Gonçalves Monteiro}

Engenheiro de produção pela Faculdade CNEC Unaí. Especialista em Engenharia da Qualidade pela Universidade Candido Mendes. Atualmente é mestrando no Programa de Pós-Graduação em Agronegócios (PROPAGA) na Universidade de Brasília e pesquisador do Núcleo de Estudos de Economia Agrícola do Ipea na Diretoria de Estudos e Políticas Regionais, Urbanas e Ambientais (Dirur). Membro do Grupo de Estudos sobre Competitividade e Sustentabilidade do Agronegócio (GECOMP/CNPq).

Email: maicongoncalves675@gmail.com

\section{Marlon Vinícius Brisola}

Pós-doutor pela Facultad de Ciencias Económicas, Universidad de Buenos Aires, Argentina, Doutor em Ciências Sociais, com especialização em estudos comparados sobre as Américas (CEPPAC/UnB), Mestre em Ciências Agrárias, com especialização em Agronegócios (UnB), Mestre em Administração de Empresas (CNEC) e Graduado em Medicina Veterinária (UFMG). Professor da Universidade de Brasília - Faculdade de Agronomia e Medicina Veterinária - Área de Ciências Sociais Aplicadas e Agronegócios. Pesquisador Credenciado do PROPAGA - Programa de Pós-graduação em Agronegócios, da UnB.

Email: mvbrisola@gmail.com

\section{Fabrício Oliveira Leitão}

Possui graduação em Administração, especialização em Gestão Educacional, mestrado em Agronegócios, e doutorado em Transportes. Atualmente é professor da Faculdade CNEC Unaí, titular das disciplinas de Teorias da Administração, Administração da Produção e Operações, Introdução à Engenharia de Produção, Desenvolvimento do Produto e Logística. Professor no UDF Centro Universitário de Brasília, lecionando as disciplinas de Gestão de Projetos, Análise e Melhoria de Processos, Arquitetura Organizacional e Gestão da Produção e Operações. Também é coordenador do curso de Engenharia de Produção da Faculdade CNEC Unaí, e supervisor da Revista CNEC com Ciência. Tem artigos publicados em Revistas Especializadas trabalhando com as linhas de pesquisa voltadas para a Gestão da Produção e Logística. É membro do grupo de pesquisa do GPIT/UnB (Grupo de Pesquisa sobre Planejamento e Gestão em Transportes) e do GECOMP/UnB (Grupo de Estudos sobre a Sustentabilidade e Competitividade do Agronegócio/UnB) onde lidera uma linha de pesquisa sobre Logística no Agronegócio. Foi consultor do Ministério da Agricultura, Pecuária e Abastecimento no projeto Suinocultura de Baixa Emissão de Carbono em 2015 e 2016.

Email: fabriciofol@hotmail.com

\section{Warley Henrique da Silva}

Doutorando em Ciências Mecânicas pelo Departamento de Engenharia Mecânica da Universidade de Brasília (UnB). Também é mestre em Agronegócios pela UnB (2017) e graduado em Engenharia de Produção (2015) com especialização em Engenharia de Segurança do Trabalho (2019) e em Engenharia de Suprimentos (2016). É professor de ensino Superior na Faculdade CNEC Unaí, onde ministra disciplinas nos cursos de Engenharia de Produção e Engenharia Civil e também coordena ambos os cursos. Também foi professor substituto do Departamento de Administração da Faculdade de Administração, Economia e Contabilidade (FACE) da UnB entre agosto de 2019 e julho de 2020, sendo responsável pelas disciplinas de Logística Organizacional e Administração de Custos.

Email: warleyhsunai@gmail.com 